\title{
FEASIBILITY STUDY OF LONGITUDINAL POLARIZATION SCHEME IN THE EXISTING CESR TUNNEL*
}

\author{
D. Wang ${ }^{\dagger}$ \\ Laboratory of Nuclear Studies, Cornell University, Ithaca, NY 14853, USA
}

\begin{abstract}
The longitudinally polarized beam is the powerful probe to study particle physics. In CESR, a high luminosity electron/positron collider operating at energy range around $5 \mathrm{GeV}(\mathrm{B}-\mathrm{mes}$ ) $)$ and possibly $1.5 \sim 2.0 \mathrm{GeV}$ (Charm physics) in coming years, the longitudinal polarization scheme is an option to be considered for the future. In this paper the feasibility of such a scheme in the existing CESR tunnel is explored. It is shown that the Siberian snake in collider ring, together with polarization gun and fast acceleration in synchrotron, is promising at low energy end of CESR operation.
\end{abstract}

\section{INTRODUCTION}

The Cornell Electron Storage Ring(CESR) has been operating at the Cornell University since 1979. The present operating energy range is 4.7 to $5.8 \mathrm{GeV}$ per beam to study the B meson family. There is a plan to extend the operation range to as low as $1.5 \mathrm{GeV}$ to study the charm physics in the coming years[1]. In future, a two-bore collider[2] is also planned to be built above the current synchrotron in the CESR tunnel to achieve very high luminosity. Our investigation is primarily based on the dual-bore collider project in which, from the polarization point of view, the situations are relatively concise compare to the single ring pretzel scheme. The major issues to realize the longitudinal polarization include: the production of polarized beams, spin rotation scheme and the control of depolarization effects.

\section{GET BEAM POLARIZED}

For the high energy storage rings, the utilization of radiative polarization (Sokolov-Ternov effect) is a natural idea. The difference in the rates for spin flip by the emission of synchrotron radiation for electrons with spins parallel or anti-parallel to the magnetic guide field can lead the build-up of the polarization of a stored electron beam. However, in the case of CESR, the major dipole field is not strong enough to provide fast build-up polarization. The bending radius of most of dipoles of synchrotron and CESR collider ring including the dualbore machine is about 80 meters. Table 1 and Table 2 show the estimated polarization time in CESR and synchrotron at their low and high operational energy range, respectively.

The build-up polarization time in major energy points is too long compare to luminosity time (about 1 hour) for collider ring and the cycle time in synchrotron $(16 \mathrm{~ms})$.

*Work is supported by the National Science Foundation

${ }^{\dagger}$ wangd@bnl.gov, present address, BNL, Upton, NY 11973
The idea that ramping beams in collider ring to high energy $(>8 \mathrm{GeV})$ to get them polarized in short time period will face two difficulties: one is the RF power (and vacuum) at high energy, one is the spin matching with spin rotator on while ramping and staying at high energy in which depolarization is strong. The use of long and strong asymmetric wigglers is restrained by the limited space in the CESR tunnel. The many short symmetric wigglers planned for CESR-Charm to adjusting the damping time and the emittance are not helpful for shortening the polarization time.

Table 1: Polarization time at low energy range of CESR and synchrotron

\begin{tabular}{|l|c|c|c|c|}
\hline Energy $(\mathrm{GeV})$ & 1.0 & 1.5 & 2.0 & 3.0 \\
\hline Pol. Time (h) & 23000 & 2570 & 715 & 95 \\
\hline
\end{tabular}

Table 2: Polarization time at high energy range of CESR and synchrotron

\begin{tabular}{|l|c|c|c|c|}
\hline Energy $(\mathrm{GeV})$ & 4.0 & 4.7 & 5.3 & 8.0 \\
\hline Pol. Time (h) & 23 & 10 & 5 & 0.7 \\
\hline
\end{tabular}

The polarized electron gun has become the mature technology in recent years and can fulfill the requirements of CESR. The polarized electron beam can be accelerated up to $300 \mathrm{MeV}$ with the existing linac. Thanks to the low dipole fields in the synchrotron the acceleration of polarized electron can be treated as proton machine at low energy. Given the fast ramping speed in the synchrotron ( $60 \mathrm{~Hz}$ repetition rate), acceleration up to $2 \mathrm{GeV}$ should be relatively easy even without compensation. To accelerate the beam to higher energy means more resonance crossings need to be overcome. The compensation techniques developed in proton machines might be necessary.

\section{SPIN ROTATIONS}

Particle physics experiments require the longitudinal polarized beam at IP therefore some kinds of spin rotation schemes must be introduced in the collider ring. Three types of spin rotators are evaluated: HERA-type minirotator, dipole-solenoid spin rotator and Siberian snake.

\subsection{HERA-type mini-rotator and dipole- solenoid rotator}

The idea of both schemes is to ensure the periodic solution of the spin vector at IP is longitudinal while 
keeping it vertical at the arc to avoid part of the depolarization effects that are particularly harmful in high energy.

The classical spin motions in magnetic fields can be described by the Thomas-BMT equation. Table 3 gives the typical bending angle and solenoid field strength needed to rotate electron spin vector by 90 degree at energies of CESR.

Table 3: To rotate spin by 90 degrees at different energy

\begin{tabular}{|c|c|c|c|c|}
\hline Energy $(\mathrm{GeV})$ & 1.55 & 2.0 & 4.7 & 5.3 \\
\hline$a \gamma$ & 3.51 & 4.53 & 10.66 & 12.03 \\
\hline Dipole (degree) & 25.7 & 19.9 & 8.4 & 7.5 \\
\hline Solenoid (T•m) & 16.2 & 20.9 & 49.2 & 55.5 \\
\hline
\end{tabular}

A single mini-rotator consists of three horizontal bends interleaved with three vertical bends. The orbit deflections produce rotations of the spins about the vertical and horizontal axes. Because the beam energy is relatively low ( $a \gamma$ is small) big deflection angles are needed. The vertical offsets can hardly be accommodated for the dual aperture machine in the already crowded CESR tunnel.

A dipole-solenoid rotator, consisting of dipoles and solenoid(s) with 90 degrees total bending angle of spin vector, has the same problem. Although its bending angles are horizontal, the long straights needed by solenoids and many quadrupoles for coupling compensation and spin matching can hardly be found in the round-like CESR tunnel. Another point is that the layouts of both kinds of rotators are energy dependent. Further spaces are required for those rotators to be workable in a range of energy. Consequently, mini-rotator and dipole-solenoid rotator can not be adopted in the existing CESR tunnel.

\subsection{Siberian Snake}

A Siberian Snake is a rotator that rotates the spin by 180 degree around an axis that lies in the horizontal plane. A periodic solution $\boldsymbol{n}_{0}(\theta+2 \pi)=\boldsymbol{n}_{0}(\theta)$ of the Thomas-BMT equation can be found in a ring with a 180 degree Siberian Snake. By installing the snake at the north IR, the vector $\boldsymbol{n}_{0}$ is longitudinal at the south IP where CLEO detector is located.

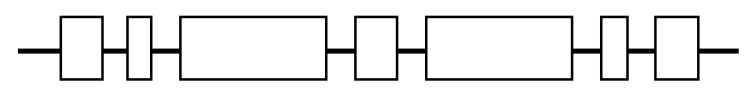

SQ1A SQ2A SOLE90 Q0 SOLE90 SQ2B SQ1B

Figure 1: Layout of Siberian Snake

The advantage of this scheme is that no movable element is required for working at a range of beam energy. However the maximum field strength determines the workable energy range of the snake. A typical design of the Siberian Snake is shown in Figure 1. The total length of the Snake optimized for low energy is $7.0 \mathrm{~m}$, which is well fit for the straight section at north IR where dual-aperture structure at arcs becomes two independent chambers and intersect each other. In case of high energy around $5 \mathrm{GeV}$ a similar design gives the $20 \mathrm{~m}$ total length.

Table 4: Parameters of elements in snake

\begin{tabular}{|c|c|c|c|c|}
\hline & S90 & SQ1 & SQ2 & Q0 \\
\hline Length(m) & 1.5 & 0.2 & 0.4 & 0.4 \\
\hline B and K & 7 Tmax & \pm 2.599 & \pm 1.9875 & 0.8916 \\
\hline
\end{tabular}

The Table 4 shows the parameters of a snake designed for low energy (up to $2 \mathrm{GeV}$ ) operation. The coupling compensation and optics optimization are included.

\section{DEPOLARIZATION EFFECTS}

In the case of the Siberian Snake, the periodic solution of spin vector at ARC is perpendicular to the major dipole field[3]. Hence it can cause very strong depolarization effects. Unlike mini-rotator and dipole-solenoid rotator, the spin matching is not applicable in practice for this scheme. Since the ultimate equilibrium polarization with snake is zero, the depolarization time becomes the key issue. According to the Derbenev-Kondratenko formula the polarization time constant to reach the equilibrium is

$$
\tau_{p}=\frac{\tau_{0}}{c+}
$$

$\tau_{0}$ is polarization build-up time without snake. C+ can be expressed as[4], in the case of the Siberian Snake,

$$
C+\approx \frac{8}{9}+\frac{11}{54}(\pi \gamma a)^{2}
$$

The Table 5 shows the depolarization time at different energies.

Table 5: C+ and depolarization time

\begin{tabular}{|l|c|c|c|c|c|}
\hline $\mathrm{E}(\mathrm{GeV})$ & 1.0 & 2.0 & 3.0 & 4.7 & 5.3 \\
\hline $\mathrm{C}+$ & 11 & 42 & 94 & 231 & 293 \\
\hline$\tau_{\mathrm{p}}$ (hours) & 2035 & 17 & 1 & 0.05 & 0.015 \\
\hline
\end{tabular}

Obviously the workable energy range for the Siberian Snake scheme is below $3 \mathrm{GeV}$.

\section{SUMMARY}

The feasibility of longitudinal polarization scheme at CESR tunnel is explored. Having considered the major relevant issues, the Siberian Snake scheme working at low energy is considered as the most promising candidate.

\section{REFERENCES}

[1] D. Rubin, these proceedings, Chicago, 2001

[2] See, for example, G. Dugan, et al., PAC'97, p318, Vancouver, 1997

[3] Y. Derbenev, A. Kondratenko, Sov. Phys. JETP 37 (1973) 968

[4] K. Yokoya, Lecture at the Advanced Accelerator School, Beijing, 1995 